\title{
SPOP accelerates acute myeloid leukemia initiation and development through miR-183-mediated METAP2 inhibition
}

\section{Jifeng Yu ( $\sim$ Dr_JifengYu@126.com )}

the First Affiliated Hospital of Zhengzhou University

\section{Yingmei Li}

First Affiliated Hospital of Zhengzhou University

\section{Ling Sun}

First Affiliated Hospital of Zhengzhou University

\section{Lijie Han}

First Affiliated Hospital of Zhengzhou University

Yu Liu

First Affiliated Hospital of Zhengzhou University

\section{Danfeng Zhang}

First Affiliated Hospital of Zhengzhou University

Haizhou Xing

First Affiliated Hospital of Zhengzhou University

\section{Xinsheng Xie}

First Affiliated Hospital of Zhengzhou University

\section{Dingming Wan}

First Affiliated Hospital of Zhengzhou University

\section{Zhongxing Jiang}

First Affiliated Hospital of Zhengzhou University

\section{Research}

Keywords: SPOP, $\beta$-catenin, miR-183, METAP2, Acute myeloid leukemia, Proliferation, Apoptosis

Posted Date: January 23rd, 2020

DOI: https://doi.org/10.21203/rs.2.21671/v1

License: (c) (1) This work is licensed under a Creative Commons Attribution 4.0 International License.

Read Full License 


\section{Abstract}

Background: Recent miRNA profiling studies have implicated the potential use of miRNAs as as diagnostic and prognostic indicators in acute myeloid leukemia (AML), which has been reportedly implicated in the interplay with certain mRNAs. Herein this study, we intend to characterize the functional relevance of SPOP/miR-183/METAP2 axis in AML in vivo and in vitro.

Methods: Differentially expressed mRNAs and downstream regulatory miRNA were predicted by in silico analysis. We induced SPOP/miR-183/METAP2 overexpression or inhibition to examine their effects on AML cell proliferation and apoptosis in vitro and tumor growth in vivo, along with their interaction with $\beta$ catenin.

Results: SPOP and miR-183 were highly expressed, while METAP2 was poorly expressed in patient peripheral blood samples and cell lines of AML. SPOP accelerated the proliferation of AML cells and repressed apoptosis. Mechanistically, SPOP enhanced $\beta$-catenin protein stability and nuclear translocation leading to upregulated expression of miR-183. MiR-183 facilitated proliferation and inhibited apoptosis of AML cells by targeting METAP2. Furthermore, miR-183 inhibition and METAP2 overexpression reversed SPOP-induced AML cell malignancy. Besides, in vitro findings were reproduced by in vivo findings.

Conclusion: SPOP stimulated AML malignant progression by inducing $\beta$-catenin stability and miR183/METAP2 axis activation, highlighting a potential therapeutic target against AML recurrence and metastasis.

\section{Background}

Acute myeloid leukemia (AML) represents a malignant disorder originating in the bone marrow, which has been recognized to cause clonal spread as well as differentiation arrest of myeloid progenitor cells [1]. It is a heterogeneous disease accompanied by a warning mortality, where the prognosis status is dependent on cytogenetic and molecular changes as well as patient-related situations, such as age, and complications [2, 3]. Furthermore, AML is a genetically heterogeneous malignant disorder of the hematopoietic system, which has been reportedly indicated to accompany with the increase of immature myeloid precursors with injured DNA [4, 5]. This aggressive hematologic neoplasm often develops to relapse resulting from drug resistance despite intensive chemotherapy [6]. Of interest, progress in unveiling the etiology of AML has extensively advanced in recent years probably due to new findings in cancer genomics and mechanistic studies of leukemogenic alterations. Notably, newly proposed molecular therapies targeting dysregulated functional pathways are currently under investigation with a few moving closer to clinical use [7].

Recently, the use of microRNAs (miRNAs)-mRNA interaction for pathogenetic studies and their potential application in clinical settings have opened a new frontier for the deciphering of therapeutic approaches for AML $[8,9]$. MiRNAs are generally deregulated in $A M L$, orchestrating pivotal genes both through direct 
targeting relationship, and through mediation of downstream factors [10]. In the present study, differentially expressed SPOP and downstream regulatory miR-183 and its target METAP2 were predicted by in silico analysis. Abnormally high expression of miR-183 has been proposed in a prior study to augment cell proliferation and cell cycle entry, but repress cell apoptosis of leukemia cells in pediatric acute myeloid leukemia [11]. The speckle-type POZ protein (SPOP) could adapt the substrate for the cullin3-RING ubiquitin ligase and fine-tune the cellular maintenance of various protein substrates, and dysregulated SPOP level and subsequent proteostatic pathway abnormality may results in the initiation of prostate and endometrial cancers [12]. Furthermore, ILF3, a substrate of SPOP, has been demonstrated to show abnormal expression in B-chronic lymphocytic leukemia [13] and to function as tumor promoter in colorectal cancer [14], suggesting SPOP-associated oncogenesis in leukemia. In addition, METAP2 has been indicated as a newly discovered anticancer molecular target [15], which exhibits anti-angiogenic activity [16]. Whereas many molecules driving metastatic growth have been identified, there is an important lack of knowledge regarding mechanisms allowing cancer initiation before expansion [17]. Herein, this study aims to delineate new mechanisms by which SPOP-mediated miR-183/METAP2 axis exerts its oncogenic effects in AML initiation and development.

\section{Materials And Methods Study subjects}

Peripheral blood samples were collected from 42 patients with AML (AML group) and 20 patients with iron deficiency anemia (normal group) admitted to the First Affiliated Hospital of Zhengzhou University from October 2018 to September 2019. There was no statistical difference in age and gender between the two groups. All the participants provided written informed consents. The study was approved by the Ethics Committee of the First Affiliated Hospital of Zhengzhou University and conducted in accordance with the Declaration of Helsinki.

\section{Cell treatment and lentiviral transduction}

Human AML cell lines (HL60, K562, U937, and KG-1) were obtained from the Shanghai Institute of Biochemistry and Cell Biology (Shanghai, China). Peripheral blood mononuclear cells (PBMCs) were extracted from peripheral blood from healthy donors as controls. Cells were incubated in RPMI containing 10\% FBS (10099141, Gibco, USA), 1\% penicillin-streptomycin (15070063, Gibco, USA), and RPMI 1640 medium (72400120, Gibco, USA) containing 200 mM L-glutamine (A2916801, Gibco, USA) [18, 19]. HEK293T cells were purchased from the American Type Culture Collection (ATCC, Manassas, VA, USA). The cells were cultured in high-sugar DMEM (1069044, Gibco, USA) supplemented with $10 \%$ FBS, $1 \%$ penicillin-streptomycin. The cells were transduced with lentiviral vectors of SPOP overexpression (oeSPOP), $\beta$-catenin overexpression (oe- $\beta$-catenin), METAP2 overexpression (oe-METAP2), shRNAs targeting SPOP (sh-SPOP-1, sh-SPOP-2), shRNAs targeting $\beta$-catenin (sh- $\beta$-catenin- 1 , sh- $\beta$-catenin- 2 ), and plasmids of miR-183 mimic, miR-183 inhibitor, as well as corresponding controls. All the lentiviruses, mimics and 
inhibitors were purchased from Sangon Biotech (Shanghai, China), which also performed the plasmid construction and lentivirus purification.

\section{In silico analysis}

The GEPIA database was employed to determine the expression of SPOP in AML, and the databases of starBase, miRDB and mirDIP to predict the downstream target mRNAs of miR-183. The processed AML transcriptome data $(n=151)$ of the TCGA database were downloaded from the UCSC xene database, and the normal sample data $(n=70)$ from the GTEX database. After the data were merged, the differential analysis was performed through the "limma" package of R language. With $|\log \mathrm{FC}|>0.5$ and FDR $<0.01$ as the selection criteria, the selected down-regulated AML-related mRNAs were intersected with the prediction results obtained from the starBase database. Finally, the expression of METAP2 was further verified in AML samples in the GEPIA database.

\section{RNA extraction and qRT-PCR}

Trizol method (16096020, Thermo Fisher Scientific, USA) was employed to extract total RNA from tissues or cells. The reverse transcription kit (B532453, Sangon Biotech, Shanghai) was adopted for miRNA and a reverse transcription kit (D7168L, Beyotime, Shanghai) for mRNAs to reverse transcribe RNA into cDNA. Primer sequences (Table 1) were designed and provided by Sangon Biotech (Shanghai, China). The miRNA (normalized to U6) or mRNA (normalized to GAPDH) levels were determined by qRT-PCR using $2^{-\triangle \triangle C T}$ method.

Table 1

Primer sequences for qRT-PCR.

\begin{tabular}{|c|c|}
\hline mRNA/miR & Sequence $\left(5^{\prime}-3^{\prime}\right)$ \\
\hline \multirow[t]{2}{*}{ SPOP } & F: TGGACCATCAATAACTTTAGC \\
\hline & R: GCCCGAACTTCACTCTTT \\
\hline \multirow[t]{2}{*}{ miR-183 } & F: GCAGTATGGCACTGGTAGA \\
\hline & R: CAGTGCGTGTCGTGGAGT \\
\hline \multirow[t]{2}{*}{ METAP2 } & F: AAAGGACAAGAATGCGAATACCC \\
\hline & R: CAGGCTTGATCCAGCTCATTAC \\
\hline \multirow[t]{2}{*}{ GAPDH } & F:GGAGCGAGATCCCTCCAAAAT \\
\hline & R:GGCTGTTGTCATACTTCTCAGG \\
\hline \multirow[t]{2}{*}{ U6 } & F: GTAATACGACTCACTATAGGGAGAAGAG \\
\hline & R: CGCGCCTGCAGGTCGAC \\
\hline F, forward; I & verse. \\
\hline
\end{tabular}




\section{Western blots}

PMSF-containing RIPA lysis buffer (P0013B, Beyotime, Shanghai, China) was employed to lyse tissues and cells to extract total protein. Nucleus and cytoplasmic protein extraction kit (P0028, Beyotime, Shanghai, China) was employed according to the instructions. Extracts were resolved by $8 \%-12 \%$ SDSPAGE. The proteins on the gel were electroblotted onto a PVDF membrane (1620177, BIO-RAD, USA), which was then blocked with $5 \%$ skim milk or $5 \%$ BSA at room temperature for $1 \mathrm{~h}$. Membranes were immunoblotted with monoclonal primary antibodies: anti-human GAPDH (ab181602, 1: 5000, Abcam, UK), anti-human Histone 3 (ab1791, 1: 5000, Abcam, UK), anti-human SPOP (ab137537, 1: 1000, Abcam, UK), anti-human $\beta$-catenin (ab227499, 1: 1000, Abcam, UK), and anti-human METAP2 (12547, 1: 1000, Cell Signaling Technology, USA) overnight at $4{ }^{\circ} \mathrm{C}$. On the next day, membranes were incubated with HRPlabeled goat anti-rabbit IgG (ab6721, 1: 5000, Abcam, UK) secondary antibody at room temperature for $1 \mathrm{~h}$. The blots were developed with an ECL reaction solution (1705062, Bio-Rad, USA) and images were captured by an Image Quant LAS 4000C gel imager (GE Healthcare, Pittsburgh, PA, USA). The total cell protein and cytoplasmic protein was normalized by GAPDH, and the nuclear protein by Histone 3.

\section{Cell viability assay by Cell Counting Kit-8 (CCK-8)}

Cell viability experiments were performed using the CCK-8 kit (GK10001, GLPBIO, USA) according to the instructions. Cells in each well were incubated with $100 \mu \mathrm{L}$ of CCK-8 reagent at room temperature. The absorbance was measured at $450 \mathrm{~nm}$ at $0 \mathrm{~h}$ At $24 \mathrm{~h}, 48 \mathrm{~h}, 72 \mathrm{~h}$, and $96 \mathrm{~h}$, and the cell proliferation curve was drawn.

\section{Cell proliferation assay by EdU staining}

The proliferation of euESC cells was assessed by an EdU Cell Proliferation Kit (C10339, Thermo Fisher Scientific, USA). The transfected cells were incubated with EdU $(50 \mu \mathrm{mol} / \mathrm{L})$ at $37^{\circ} \mathrm{C}$ for $6 \mathrm{~h}$, and then stained with Hoechst 33342, followed by observation and photographing under a microscope (BX63, Olympus, Japan). The ratio of EdU-positive cells to Hoechst-positive cells was employed to estimate the EdU positive rate.

\section{Cell proliferation assay by Ki67 immunohistochemistry in tissues}

The solid tumor tissue sections were subjected to $3 \% \mathrm{H}_{2} \mathrm{O}_{2}$ to block endogenous peroxidase. Antigen retrieval was conducted by citric acid buffer in the microwave. The sections were incubated with normal goat serum blocking solution (Sangon Biotech Co., Ltd., Shanghai, China) at room temperature for 20 min. Next, sections were treated with diluted primary rabbit anti-mouse Ki67 antibody (ab15580, 1: 500, Abcam, UK) at $4{ }^{\circ} \mathrm{C}$ overnight, followed by incubation with HRP-labeled goat anti-rabbit lgG secondary antibody (ab6721, 1: 5000, Abcam, UK). After treatment with SABC, sections were developed using a DAB color development kit (Sigma, USA). Following staining in hematoxylin, sections were observed under a microscope to count the Ki67 positive cell rate. 


\section{Flow cytometric analysis of cell apoptosis}

Cell apoptosis was examined using an Annexin V-FITC and propidium iodide (PI) kit (APOAF, SigmaAldrich, USA). Cells were treated differently and cultured for $48 \mathrm{~h}$. The collected cells were incubated with $10 \mu \mathrm{L}$ Annexin V-FITC and $5 \mu \mathrm{L}$ PI for 15 min at room temperature without light exposure. A flow cytometer (Becton Dickinson, USA) was employed to examine the cell apoptosis and calculate the apoptosis rate.

\section{Cell apoptosis assay by TUNEL staining in tissues}

An apoptosis detection kit (C1098, Beyotime, Shanghai, China) was employed for TUNEL assay in tissues. The sections were subjected to DNase-free proteinase $\mathrm{K}$ treatment at $37^{\circ} \mathrm{C}$ for $15 \mathrm{~min}$. Then, sections were stained in TUNEL solution at $37^{\circ} \mathrm{C}$ for $60 \mathrm{~min}$ without light exposure. After being mounted with DAPI medium, sections were observed under a microscope to count the apoptosis rate.

\section{Luciferase activity assay}

The miR-183 promoter sequence (miR-183 WT) and mutation sequence (miR-183 Mut) that bind to $\beta$ catenin were synthesized. METAP2 mRNA 3'UTR sequence (METAP2 WT) and mutation sequence (METAP2 Mut) that bind to miR-183 were introduced into pGL3-basic vector (E1751, Promega, USA). The target fragment was inserted into pGL3 vector using T4 DNA ligase (M0204S, New England Biolabs, USA) after restriction digestion. Renilla luciferase plasmid and the constructed luciferase reporter plasmid were co-transfected with oe-NC, oe- $\beta$-catenin or mimics-NC, miR-183 mimics into HEK293T cells for $48 \mathrm{~h}$ transfection. The Dual-Luciferase ${ }^{\circledR}$ Repeat Assay System Kit (E1910, Promega, USA) was employed for assessing luciferase activity on the GloMax ${ }^{\circledR}$ 20/20 Luminometer (E5311, Promega, US). Relative luciferase activity = firefly luciferase activity/Renilla luciferase activity. All vectors were constructed by Sangon Biotech Co., Ltd., Shanghai, China.

\section{ChIP assay}

ChIP assays were performed using the EZ-Magna ChIP kit (EMD Millipore, USA). K562 and KG-1 cells were fixed with $1 \%$ paraformaldehyde and incubated with glycine for $10 \mathrm{~min}$ to produce DNA-protein cross-links. Cells were then lysed using cell lysis buffer and nuclear lysis buffer, and sonicated to produce 200-300 bp chromatin fragments. Next, the lysate was immunoprecipitated with magnetic protein A beads binding to the antibody. The rabbit IgG (ab172730, 1: 100, Abcam, UK) was added to the negative control, and $\beta$-catenin antibody (ab227499, 1: 1000, Abcam, UK) was added to the Anti- $\beta$-catenin group. Finally, miR-183 promoter fragment in the precipitate was evaluated by qRT-PCR. miR-183 CHIP primer (F: 5'-GTCCTCTGGGGAGATAGACC-3', R: 5'-ACACTGCTTAATGCTCCACCC-3').

\section{Immunofluorescence staining}

The cells were fixed with $4 \%$ paraformaldehyde, and blocked with $5 \%$ goat serum (diluted with $0.3 \%$ PBST) for $1 \mathrm{~h}$ at room temperature. Cells were incubated with primary antibody rabbit anti-human $\beta$ catenin (ab227499, 1: 1000, Abcam, UK) diluted in blocking solution at room temperature for $1 \mathrm{~h}$. Then 
cells were incubated with red fluorescent protein (Cy3) labeled goat anti-rabbit secondary antibody IgG H\&L (ab6939, 1: 500, Abcam, UK) at room temperature for $1 \mathrm{~h}$. After being mounted with DAPI medium, sections were observed under a microscope.

\section{Xenografts in nude mice}

Totally, 48 male nude mice (4-6 weeks of age, weighing 15-18 g) were purchased from the Experimental Animal Center of Zhengzhou University and randomly divided into 8 groups $(n=6)$. K562 cells and KG-1 cells were respectively transduced with oe-NC, oe-NC + oe-METAP2, oe-SPOP + oe-NC, or oe-SPOP + oeMETAP2. The nude mice was subcutaneously injected at the right side with about $10^{7}$ lentivirus-infected K562 cells or KG-1 cells. The tumor volume formed was measured with a micrometer every 5 days. All mice were sacrificed 1 month later, and tumors were removed and weighed [18, 20].

\section{Statistical analysis}

All data are shown as mean \pm standard deviation and analyzed by SPSS 21.0 software (IBM, Armonk, NY, USA), with $p<0.05$ as a level of statistically significance. Comparison of unpaired data with normal distribution and equal variance was conducted using unpaired t test. Comparisons among multiple groups were conducted using one-way analysis of variance (ANOVA) with Tukey's post hoc test.

Statistical analysis in relation to time-based measurements within each group was performed with the use of repeated measures ANOVA, followed by a Bonferroni's post-hoc test.

\section{Results}

\section{SPOP overexpression accelerates AML cell proliferation and inhibits apoptosis}

Analysis of GEPIA database revealed that SPOP was up-regulated in AML $(p<0.05$, Fig. 1A). Furthermore, the expression of SPOP in the peripheral blood of AML group was higher than that in the normal group ( $p$ $<0.05$, Fig. 1B, C). Therefore, we speculate that SPOP may mediate cell activities in AML. To verify this hypothesis, we first examined the expressions of SPOP in four AML cell lines (HL60, K562, U937, and KG1). The expression of SPOP in K562, U937, and KG-1 cells was higher than that in PBMC cells $(p<0.05$, Fig. 1D, E). We selected K562 and KG-1 cells for subsequent experiments. The efficiency of overexpressing or silencing SPOP in K562 and KG-1 cells was validated. The results manifested that the expression of SPOP in the oe-SPOP group was higher than that in the oe-NC group, and that in the shSPOP-1 and sh-SPOP-2 groups was lower than that of the sh-NC group $(p<0.05$, Fig. $1 F, G)$. The sh-SPOP1 group (sh-SPOP group) was selected for subsequent experiments, due to its stronger silencing efficiency than the sh-SPOP-2 group.

CCK-8 assay identified increased cell viability in response to oe-SPOP treatment, and lowered cell viability to sh-SPOP treatment $(\mathrm{Fig} .1 \mathrm{H})$. EdU assay manifested that EdU-positive cells was increased in response to oe-SPOP treatment, along with lowered cell proliferation in response to sh-SPOP treatment (Fig. 1I). 
Flow cytometric data revealed attenuated AML cell apoptosis in response to oe-SPOP treatment, and that in response to sh-SPOP treatment was facilitated (Fig. 1J). Together, SPOP overexpression accelerates the proliferation of AML cells and inhibits their apoptosis.

\section{SPOP enhances $\beta$-catenin protein expression and nuclear translocation leading to elevated miR-183 expression}

Prior evidence proposed that $\beta$-catenin can facilitate the occurrence of AML, and SPOP can indirectly enhance the stability and nuclear translocation of $\beta$-catenin in the cytoplasm in renal cancer cells [21, 22]. Therefore, we speculate that in AML cells, SPOP may also stabilize $\beta$-catenin expression and nuclear translocation in the cytoplasm. To verify this hypothesis, we first tested $\beta$-catenin expression in K562 and KG-1 cells. The treatment of oe-SPOP resulted in elevated total $\beta$-catenin protein level, and cytoplasmic and nuclear levels, while $\beta$-catenin protein level was diminished in the presence of sh-SPOP transduction (Fig. 2A). Immunofluorescence assay found increased $\beta$-catenin distribution in the nucleus and cytoplasm following treatment of oe-SPOP (Fig. 2B). Thus, in AML cells, SPOP can augment the stability of cytoplasm and nuclear translocation of $\beta$-catenin.

MiR-183 has been reportedly suggested to highly express in AML and stimulate the occurrence of this disease [18]. Besides, $\beta$-catenin and TCF bind to the miR-183 promoter region to enhance the miR-183 expression in liver cancer cells [23]). Therefore, we speculate that the high expression of miR-183 in AML cells may also be regulated by $\beta$-catenin. First, we found that the expression of miR-183 in peripheral blood of the AML group was higher than that in the normal group ( $p<0.05$, Fig. $2 \mathrm{C})$. Next, we assessed the efficiency of overexpressing and silencing $\beta$-catenin. The expression of $\beta$-catenin was increased upon treatment of oe- $\beta$-catenin, and reduced upon treatment of sh- $\beta$-catenin- 1 and sh- $\beta$-catenin-2 (Fig. 2D, E). The sh- $\beta$-catenin-1 (sh- $\beta$-catenin group) with stronger silencing efficiency than sh- $\beta$-catenin- 2 was selected for subsequent experiments. oe- $\beta$-catenin was co-transfected into HEK293T cells with miR-183 Wt and miR-183 Mut, respectively. Western blot assay revealed in the presence of miR-183 Wt and miR183 Mut, the $\beta$-catenin expression was enhanced upon oe- $\beta$-catenin transduction versus oe-NC treatment $(p<0.05)$. The dual luciferase report assay suggested that the luciferase activity in the miR-183 Wt group increased following addition of oe- $\beta$-catenin, while the luciferase activity in the miR-183 Mut group did not change (Fig. 2F). The CHIP assay manifested that relative to IgG, $\beta$-catenin enrichment in the miR-183 promoter region was elevated, and the enrichment was facilitated after overexpressing $\beta$-catenin (Fig. 2G). In addition, we unraveled that miR-183 expression was elevated by oe-SPOP and oe- $\beta$-catenin, and reduced by sh-SPOP and sh- $\beta$-catenin. Whereas, combined treatment of oe-SPOP + sh- $\beta$-catenin showed lower miR-183 expression than oe- $\beta$-catenin treatment, and combined treatment of sh-SPOP + oe$\beta$-catenin showed higher miR-183 expression than sh-SPOP treatment (Fig. $2 \mathrm{H}$ ). These results indicate that SPOP accelerates $\beta$-catenin protein expression and nuclear translocation, thus elevating miR-183 expression. 


\section{MiR-183 overexpression enhances proliferation and inhibits apoptosis of AML cells by targeting METAP2}

In order to further clarify the downstream mechanism of miR-183, we, through bioinformatic analysis, we obtained 8890 up-regulated and 7791 down-regulated mRNAs (Fig. 3A), and intersected the mRNAs from different databases (Fig. 3B). In these four databases, 12 genes (GCLM, GNG12, METAP2, EXO1, TET1, RHOBTB1, YOD1, SMCO4, XPOT, GTF2H1, SPATS2, LRP6) were found in the intersection. No previous studies could be found on target relationship between miR-183 and METAP2. The GEPIA database analysis showed that METAP2 was down-regulated in AML samples $(p<0.05$, Fig. $3 C)$. Moreover, our experimental data manifested that the expression of METAP2 in the AML group was lower than that in the Normal group $(p<0.05$, Fig. 3D, E). The binding sites between miR-183 and METAP2 were predicted by Starbase database (Fig. 3F).

In KG-1 and K562 cells, miR-183 mimics treatment resulted in increased miR-183 expression, and miR-183 inhibitor treatment resulted in diminished miR-183 expression (Fig. $3 \mathrm{G}$ ). In the presence of METAP2 WT and METAP2 Mut, the expression of miR-183 was elevated in response to miR-183 mimics $(p<0.05)$. The dual luciferase report assay manifested that the luciferase activity in the METAP2 WT group was reduced upon miR-183 mimics treatment, and the luciferase activity didn't differ appreciably in the METAP2 Mut group (Fig. 3H). Moreover, we found that, in KG-1 and K562 cells, the expression of METAP2 was lowered following miR-183 mimics treatment, and which was elevated in response to miR-183 inhibitor treatment (Fig. $3 \mathrm{l}, \mathrm{J}$ ). The above data indicate that miR-183 targets and inhibits METAP2 expression.

Next, we further investigated whether miR-183 can expedite the proliferation and halt apoptosis of AML cells by inhibiting METAP2 expression. First, the experimental data suggested that miR-183 mimics led to miR-183 upregulation, and combined treatment of miR-183 mimics + oe-METAP2 resulted in higher miR183 expression than oe-METAP2 alone did (Fig. 3K). Besides, miR-183 mimics led to reduced METAP2 expression, and combined treatment of miR-183 mimics + oe-METAP2 resulted in higher METAP2 expression than miR-183 mimics alone did (Fig. 3K, L). CCK-8 assay found that oe-METAP2 suppressed cell viability, and miR-183 mimics enhanced cell viability, whereas, combined treatment of miR-183 mimics + oe-METAP2 resulted in impeded cell viability induced by miR-183 mimics (Fig. 3M). EdU assay revealed that oe-METAP2 diminished EdU-positive cells, and miR-183 mimics elevated EdU-positive cells, whereas, combined treatment of miR-183 mimics + oe-METAP2 resulted in reduced EdU-positive cells that were increased by miR-183 mimics (Fig. 3N). Flow cytometric data manifested that oe-METAP2 accelerated cell apoptosis, and miR-183 mimics repressed cell apoptosis, whereas, combined treatment of miR-183 mimics + oe-METAP2 resulted in enhanced cell apoptosis that was suppressed by miR-183 mimics (Fig. 30).

Together, miR-183 targets and negatively regulates METAP2 expression, thereby promoting the proliferation and inhibiting apoptosis of AML cells. 


\section{MiR-183 inhibition represses SPOP-induced proliferation of AML cells}

Next, we further investigated whether miR-183 can restrict the SPOP overexpression-induced proliferation of AML cells. First, we found that the expression of SPOP and $\beta$-catenin was elevated in response to treatment of oe-SPOP + inhibitor-NC or oe-SPOP + miR-183 inhibitor, relative to treatment of oe-NC + inhibitor-NC or oe-NC + miR-183 inhibitor $(\mathrm{p}<0.05)$. Besides, miR-183 expression was lower following oe$\mathrm{NC}+$ miR-183 inhibitor treatment, relative to oe-NC + inhibitor-NC treatment $(\mathrm{p}<0.05)$. The treatment of oe$\mathrm{SPOP}+$ inhibitor-NC stimulated miR-183 expression versus oe-NC + inhibitor-NC treatment $(p<0.05)$. Moreover, combined treatment of oe-SPOP + miR-183 inhibitor reduced miR-183 expression versus oeSPOP + inhibitor-NC treatment $(p<0.05)($ Fig. 4A, B).

CCK-8 assay manifested that miR-183 inhibitor attenuated cell viability and oe-SPOP enhanced cell viability, but the combined treatment of oe-SPOP and miR-183 inhibitor appreciably repressed the promotion of cell viability induced by oe-SPOP (Fig. 4C). Additionally, EdU assay demonstrated that miR183 inhibitor diminished EdU-positive cells and oe-SPOP elevated EdU-positive cells, but the combined treatment of oe-SPOP and miR-183 inhibitor appreciably diminished the increase in EdU-positive cells induced by oe-SPOP (Fig. 4D). Besides, flow cytometric results manifested that miR-183 inhibitor facilitated cell apoptosis and oe-SPOP restricted cell apoptosis, but the combined treatment of oe-SPOP and miR-183 inhibitor appreciably augmented the cell apoptosis that was repressed by oe-SPOP (Fig. 4E).

Therefore, inhibiting miR-183 can reverse SPOP-induced AML cell proliferation enhancement and apoptosis suppression.

\section{METAP2 overexpression restricts SPOP-induced proliferation of AML cells}

Next, we sought to assess whether METAP2 can restrict the SPOP overexpression-induced proliferation of AML cells. The oe-SPOP + oe-NC treatment and oe-SPOP + oe-METAP2 treatment showed higher expression of SPOP, $\beta$-catenin and miR-183 than the oe-NC treatment and oe-NC + oe-METAP2 treatment $(p<0.05)$. The oe-NC + oe-METAP2 treatment resulted in higher METAP2 expressions, relative to oe-NC treatment $(p<0.05)$. The expression of METAP2 was reduced upon the oe-SPOP + oe-NC treatment, relative to oe-NC treatment $(p<0.05)$, and the expression of METAP2 following the oe-SPOP + oe-METAP2 treatment was higher than that in response to oe-SPOP + oe-NC $(p<0.05$, Fig. 5A, B).

CCK-8 assay suggested that oe-METAP2 led to lowered cell viability, and oe-SPOP enhanced cell viability, but the combined treatment of oe-METAP2 and oe-SPOP appreciably repressed the promotion of cell viability induced by oe-SPOP (Fig. 5C). Additionally, EdU assay demonstrated that oe-METAP2 diminished EdU-positive cells and oe-SPOP elevated EdU-positive cells, but the combined treatment of oe-SPOP and oe-METAP2 appreciably diminished the increase in EdU-positive cells induced by oe-SPOP (Fig. 5D). Besides, flow cytometric results manifested that oe-METAP2 facilitated cell apoptosis and oe-SPOP 
restricted cell apoptosis, but the combined treatment of oe-SPOP and oe-METAP2 appreciably augmented the cell apoptosis that was repressed by oe-SPOP (Fig. 5E).

Together, overexpression of METAP2 can reverse SPOP-induced AML cell proliferation enhancement and apoptosis suppression.

\section{SPOP facilitates AML initiation by mediating $\beta$-catenin expression and the miR-183/METAP2 axis in vivo}

Next, we further measured xenograft tumors in nude mice to verify that SPOP affects the growth of AML cells by mediating $\beta$-catenin expression and the miR-183/METAP2 axis in vivo. The tumor volume and weight were reduced in response to oe-METAP2 treatment, and they were elevated in response to oe SPOP. Whereas, the combined treatment of oe-SPOP and oe-METAP2 appreciably repressed the tumor growth induced by oe-SPOP (Fig. 6A). Moreover, the expression of SPOP, $\beta$-catenin and METAP2 in the xenograft tumors was higher following treatment of oe-SPOP + oe-NC and oe -SPOP + oe-METAP2, relative to treatment of oe-NC and oe-NC + oe-METAP2 $(p<0.05)$. The oe-METAP2 treatment resulted in increased expression of METAP2, and the oe-SPOP treatment diminished expression of METAP2. Whereas, the combined treatment of oe-SPOP and oe-METAP2 appreciably enhanced the expression of METAP2 that was suppressed by oe-SPOP (Fig. 6B, C).

Ki67 immunohistochemical analysis manifested that oe-METAP2 diminished Ki67-positive cells and oeSPOP elevated Ki67-positive cells, but the combined treatment of oe-SPOP and oe-METAP2 appreciably diminished the increase in Ki67-positive cells induced by oe-SPOP (Fig. 6D). Besides, TUNEL staining manifested that oe-METAP2 facilitated cell apoptosis and oe-SPOP restricted cell apoptosis, but the combined treatment of oe-SPOP and oe-METAP2 appreciably augmented the cell apoptosis that was repressed by oe-SPOP (Fig. 6E).

Together, SPOP affects the occurrence and growth of AML by mediating the expression of $\beta$-catenin and regulating the activation of miR-183/METAP2 axis.

\section{Discussion}

Current therapies, such as chemotherapy and allogeneic stem cell transplantation, for AML are commonly effective a limited population of younger and fit patients, but the majority of elder individuals suffer from unfavorable prognosis and low survival [1,24]. Well-documented evidence exists proving that multiple miRNAs have a critical role to play in orchestrating leukemia cell proliferation and apoptosis, which may potentially be used as therapeutic biomarkers $[25,26]$. In this current study, the objective was to illuminate the molecular mechanism by which the SPOP-mediated miR-183/METAP2 axis fine-tunes the malignant phenotypes of AML cells (Fig. 7). 
The experimental data unveiled that SPOP and miR-183 were highly expressed, while METAP2 was poorly expressed in patient peripheral blood samples and cell lines of AML. SPOP overexpression accelerated AML cell proliferation and suppressed apoptosis. SPOP has been acknowledged as a substrate adaptor of the CUL3 ubiquitin ligase complex, which functions as a potential inhibitor of fetal hemoglobin [27]. The oncogenic function of SPOP has been identified in prior studies. For example, abnormally high SPOP expression has been identified in the cytoplasm of clear-cell renal cell carcinoma, which was shown to expedite cancer cell proliferation consequently augmenting renal tumorigenesis [28]. Silencing of SPOP results in strengthened kidney cancer cell apoptosis through orchestrating ubiquitination and degradation of key mRNAs controlling cellular proliferation and apoptosis [29]. Of interest, the oncogenic SPOP functionally associates with tumor suppressor SETD2 via controlling its protein stability and gene expression [30].

However, in this study, we unmasked mechanistically that the oncogenic role of SPOP was realized through enhancing $\beta$-catenin protein expression and nuclear translocation, which elevated miR-183 expression. The enhancement that SPOP triggered in $\beta$-catenin protein expression as well as its nuclear translocation has been demonstrated previously in renal cancer [22]. Moreover, the oncogenic function of miR-183 in hepatocellular carcinoma was reported to be triggered by $\beta$-catenin activation, and silencing of CTNNB1 ( $\beta$-catenin) could diminish expression of miR-183 [23]. In addition, the Wnt/ $\beta$-catenin signaling activation has been highlighted to be implicated in the pathogenesis of AML due to its stimulation on cell cycle progression and suppression on apoptosis of AML cells [31]. Inhibition of $\beta$ catenin leads to concordantly restricted tumorigenic potential in AML [32]. It is clear that SPOP-substrate protein interaction associates with the oncogenic SPOP-signaling pathways [28]. These evidences verify the pivotal function of miR-183 in the oncogenic SPOP role in AML.

Next, we further extended our understanding mechanistically by revealing that miR-183 specifically targeted and inversely regulated METAP2 expression. MiR-183 overexpression enhanced proliferation and repressed apoptosis of AML cells by targeting METAP2. METAP2 has been shown to exert antiangiogenic activity and to conduct actions toward tumor suppression [15]. Further, the study of Frottin et al. documented that METAP2 could stimulate cell selectivity for a potent anti-cancer agent in synergy [33]. Moreover, we also carried a wide array of assays to validate that miR-183 inhibition and METAP2 overexpression could repress SPOP-induced proliferation and augment SPOP-inhibited apoptosis of AML cells. The in vivo experiments consistently validated that SPOP facilitated AML initiation by mediating $\beta$ catenin expression and the miR-183/METAP2 axis.

\section{Conclusion}

Thus, we strongly feel based on the evidence of this study that therapeutic strategies should be directed towards the down-regulation of SPOP and miR-183, which may potentially be a clinically viable targets in the treatment of AML. The oncogenic SPOP accelerated tumorigenesis and development of AML through miR-183-mediated METAP2 inhibition. As we further elucidate the epigenetic regulatory mechanisms underlying $\mathrm{AML}$, there is great potential for translational applications for patients. 


\section{Abbreviations}

Acute myeloid leukemia (AML)

microRNAs (miRNAs)

speckle-type POZ protein (SPOP)

Peripheral blood mononuclear cells (PBMCs)

overexpression (oe-METAP2)

propidium iodide $(\mathrm{PI})$

\section{Declarations}

\section{Acknowledgeable}

We give our sincere gratitude to the reviewers for their valuable suggestions.

\section{Author contributions}

Jifeng Yu , Yingmei Li and Ling Sun designed the study. Lijie Han, Yu Liu, Danfeng Zhang, Haizhou Xing and Xinsheng Xie collected the data, carried out data analyses and produced the initial draft of the manuscript. Jifeng Yu, Dingming Wan and Zhongxing Jian contributed to drafting the manuscript. All authors have read and approved the final submitted manuscript.

\section{Funding}

This study was supported by the Key Scientific Research Project of Henan Provincial Education Department (20A320062 and 19A320046), National Natural Science Foundation of China (U1804192) and Special Talents Project Fund of the First Affiliated Hospital of Zhengzhou University, Zhengzhou, China.

\section{Ethics approval and consent to participate}

This study was approved by the Ethics Committee of The First Affiliated Hospital of Zhengzhou University and the methods were carried out in accordance with the approved guidelines. All the patients have been informed and signed informed consent before the experiments.

\section{Availability of data and materials}

The datasets generated/analysed during the current study are available 
All authors consent for publication.

\section{Conflicts of interests}

None

\section{References}

1. Shallis RM, Wang R, Davidoff A, Ma X, Zeidan AM. Epidemiology of acute myeloid leukemia: Recent progress and enduring challenges. Blood Rev. 2019;36(70-87.

2. Paul S, Rausch CR, Jabbour EJ. The face of remission induction. Br J Haematol. 2020;188(1):101-15.

3. Hansrivijit P, Gale RP, Barrett J, Ciurea SO. Cellular therapy for acute myeloid Leukemia - Current status and future prospects. Blood Rev. 2019;37(100578.

4. Xu R, Yu S, Zhu D, Huang X, Xu Y, Lao Y, Tian Y, Zhang J, Tang Z, Zhang Z, et al. hCINAP regulates the DNA-damage response and mediates the resistance of acute myelocytic leukemia cells to therapy. Nat Commun. 2019;10(1):3812.

5. Yu J, Li Y, Li T, Li Y, Xing H, Sun H, Sun L, Wan D, Liu Y, Xie X, et al. Gene mutational analysis by NGS and its clinical significance in patients with myelodysplastic syndrome and acute myeloid leukemia. Exp Hematol Oncol. 2020;9(2.

6. Stief SM, Hanneforth AL, Weser S, Mattes R, Carlet M, Liu WH, Bartoschek MD, Dominguez Moreno H, Oettle $\mathrm{M}, \mathrm{Kempf} \mathrm{J}$, et al. Loss of KDM6A confers drug resistance in acute myeloid leukemia. Leukemia. 2020;34(1):50-62.

7. Basheer FT, Vassiliou GS. Genome-scale drop-out screens to identify cancer cell vulnerabilities in AML. Curr Opin Genet Dev. 2019;54(83-7.

8. Ganesan S, Palani HK, Lakshmanan V, Balasundaram N, Alex AA, David S, Venkatraman A, Korula A, George B, Balasubramanian P, et al. Stromal cells downregulate miR-23a-5p to activate protective autophagy in acute myeloid leukemia. Cell Death Dis. 2019;10(10):736.

9. Liu B, Ma X, Liu Q, Xiao Y, Pan S, Jia L. Aberrant mannosylation profile and FTX/miR-342/ALG3-axis contribute to development of drug resistance in acute myeloid leukemia. Cell Death Dis. 2018;9(6):688.

10. Schneider E, Pochert N, Ruess C, MacPhee L, Escano L, Miller C, Krowiorz K, Delsing Malmberg E, Heravi-Moussavi A, Lorzadeh A, et al. MicroRNA-708 is a novel regulator of the Hoxa9 program in myeloid cells. Leukemia. 2019;

11. Wang X, Zuo D, Yuan Y, Yang X, Hong Z, Zhang R. MicroRNA-183 promotes cell proliferation via regulating programmed cell death 6 in pediatric acute myeloid leukemia. J Cancer Res Clin Oncol. 2017;143(1):169-80.

12. Cuneo MJ, Mittag T. The ubiquitin ligase adaptor SPOP in cancer. FEBS J. 2019;286(20):3946-58.

13. Agnoletto C, Brunelli L, Melloni E, Pastorelli R, Casciano F, Rimondi E, Rigolin GM, Cuneo A, Secchiero P, Zauli G. The anti-leukemic activity of sodium dichloroacetate in p53mutated/null cells is mediated 
by a p53-independent ILF3/p21 pathway. Oncotarget. 2015;6(4):2385-96.

14. Li K, Wu JL, Qin B, Fan Z, Tang Q, Lu W, Zhang H, Xing F, Meng M, Zou S, et al. ILF3 is a substrate of SPOP for regulating serine biosynthesis in colorectal cancer. Cell Res. 2019;

15. Datta B. Roles of P67/MetAP2 as a tumor suppressor. Biochim Biophys Acta. 2009;1796(2):281-92.

16. Weglarz-Tomczak E, Talma M, Giurg M, Westerhoff HV, Janowski R, Mucha A. Neutral metalloaminopeptidases APN and MetAP2 as newly discovered anticancer molecular targets of actinomycin D and its simple analogs. Oncotarget. 2018;9(50):29365-78.

17. Forte D, Krause DS, Andreeff M, Bonnet D, Mendez-Ferrer S. Updates on the hematologic tumor microenvironment and its therapeutic targeting. Haematologica. 2019;104(10):1928-34.

18. Zheng Z, Zheng X, Zhu Y, Gu X, Gu W, Xie X, Hu W, Jiang J. miR-183-5p Inhibits Occurrence and Progression of Acute Myeloid Leukemia via Targeting Erbin. Mol Ther. 2019;27(3):542-58.

19. Vaz C, Ahmad HM, Sharma P, Gupta R, Kumar L, Kulshreshtha R, Bhattacharya A. Analysis of microRNA transcriptome by deep sequencing of small RNA libraries of peripheral blood. BMC Genomics. 2010;11(288.

20. Wang A, Wu H, Chen C, Hu C, Qi Z, Wang W, Yu K, Liu X, Zou F, Zhao Z, et al. Dual inhibition of AKT/FLT3-ITD by A674563 overcomes FLT3 ligand-induced drug resistance in FLT3-ITD positive AML. Oncotarget. 2016;7(20):29131-42.

21. Xin H, Li C, Wang M. DIXDC1 promotes the growth of acute myeloid leukemia cells by upregulating the Wnt/beta-catenin signaling pathway. Biomed Pharmacother. 2018;107(1548-55.

22. Zhao W, Zhou J, Deng Z, Gao Y, Cheng Y. SPOP promotes tumor progression via activation of betacatenin/TCF4 complex in clear cell renal cell carcinoma. Int J Oncol. 2016;49(3):1001-8.

23. Leung WK, He M, Chan AW, Law PT, Wong N. Wnt/beta-Catenin activates MiR-183/96/182 expression in hepatocellular carcinoma that promotes cell invasion. Cancer Lett. 2015;362(1):97-105.

24. Yu J, Li Y, Xing H, Pan Y, Sun H, Wan D, Liu Y, Xie X, Wang C, Sun L, et al. Clinical Characteristics And Outcome Of Biphenotypic Acute Leukemia: 10 Case Reports And Literature Review. Cancer Manag Res. 2019;11(9297-306.

25. Liu Y, Cheng Z, Pang Y, Cui L, Qian T, Quan L, Zhao H, Shi J, Ke X, Fu L. Role of microRNAs, circRNAs and long noncoding RNAs in acute myeloid leukemia. J Hematol Oncol. 2019;12(1):51.

26. Jifeng Yu* YL, Yue Pan, Yu Liu, Haizhou Xing, Xinsheng Xie, Dingming Wan, Zhongxing Jiang. Deficient regulatory innate lymphoid cells and differential expression of miRNAs in acute myeloid leukemia quantified by next generation sequence. Cancer Management and Research. 2019;11(10969-82.

27. Lan X, Khandros E, Huang P, Peslak SA, Bhardwaj SK, Grevet JD, Abdulmalik O, Wang H, Keller CA, Giardine B, et al. The E3 ligase adaptor molecule SPOP regulates fetal hemoglobin levels in adult erythroid cells. Blood Adv. 2019;3(10):1586-97.

28. Guo ZQ, Zheng T, Chen B, Luo C, Ouyang S, Gong S, Li J, Mao LL, Lian F, Yang Y, et al. Small-Molecule Targeting of E3 Ligase Adaptor SPOP in Kidney Cancer. Cancer Cell. 2016;30(3):474-84. 
29. Li G, Ci W, Karmakar S, Chen K, Dhar R, Fan Z, Guo Z, Zhang J, Ke Y, Wang L, et al. SPOP promotes tumorigenesis by acting as a key regulatory hub in kidney cancer. Cancer Cell. 2014;25(4):455-68.

30. Zhu K, Lei PJ, Ju LG, Wang X, Huang K, Yang B, Shao C, Zhu Y, Wei G, Fu XD, et al. SPOP-containing complex regulates SETD2 stability and H3K36me3-coupled alternative splicing. Nucleic Acids Res. 2017;45(1):92-105.

31. Yuan Y, Wang Q, Ma SL, Xu LQ, Liu MY, Han B, Du N, Sun XL, Yin XL, Cao FF. IncRNA PCAT-1 interacting with FZD6 contributes to the malignancy of acute myeloid leukemia cells through activating Wnt/beta-catenin signaling pathway. Am J Transl Res. 2019;11(11):7104-14.

32. Xiao H, Cheng Q, Wu X, Tang Y, Liu J, Li X. ADAR1 may be involved in the proliferation of acute myeloid leukemia cells via regulation of the Wnt pathway. Cancer Manag Res. 2019;11(8547-55.

33. Frottin F, Bienvenut WV, Bignon J, Jacquet E, Vaca Jacome AS, Van Dorsselaer A, Cianferani S, Carapito C, Meinnel T, Giglione C. MetAP1 and MetAP2 drive cell selectivity for a potent anti-cancer agent in synergy, by controlling glutathione redox state. Oncotarget. 2016;7(39):63306-23.

\section{Figures}
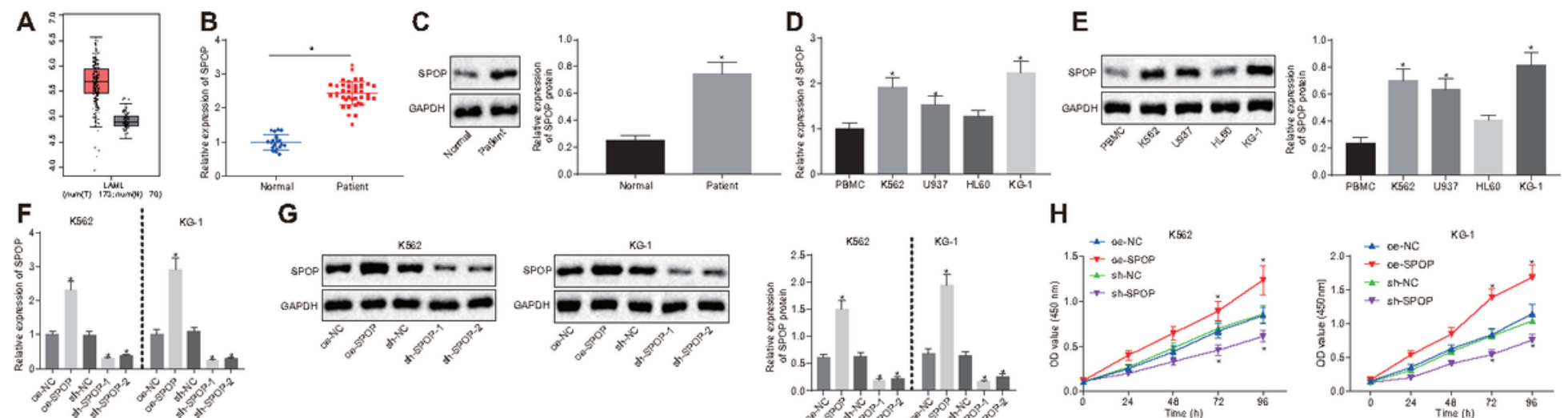

G
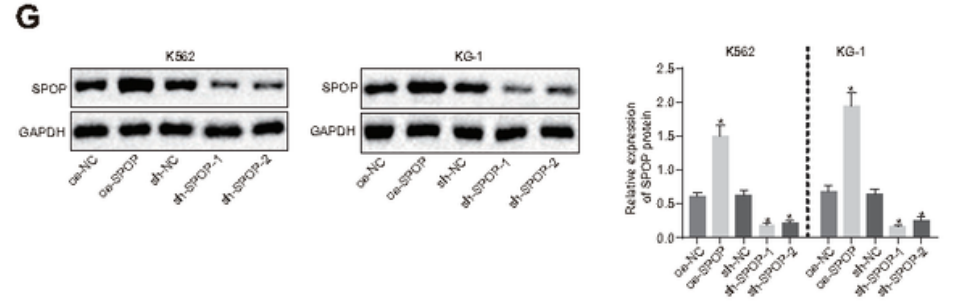

H
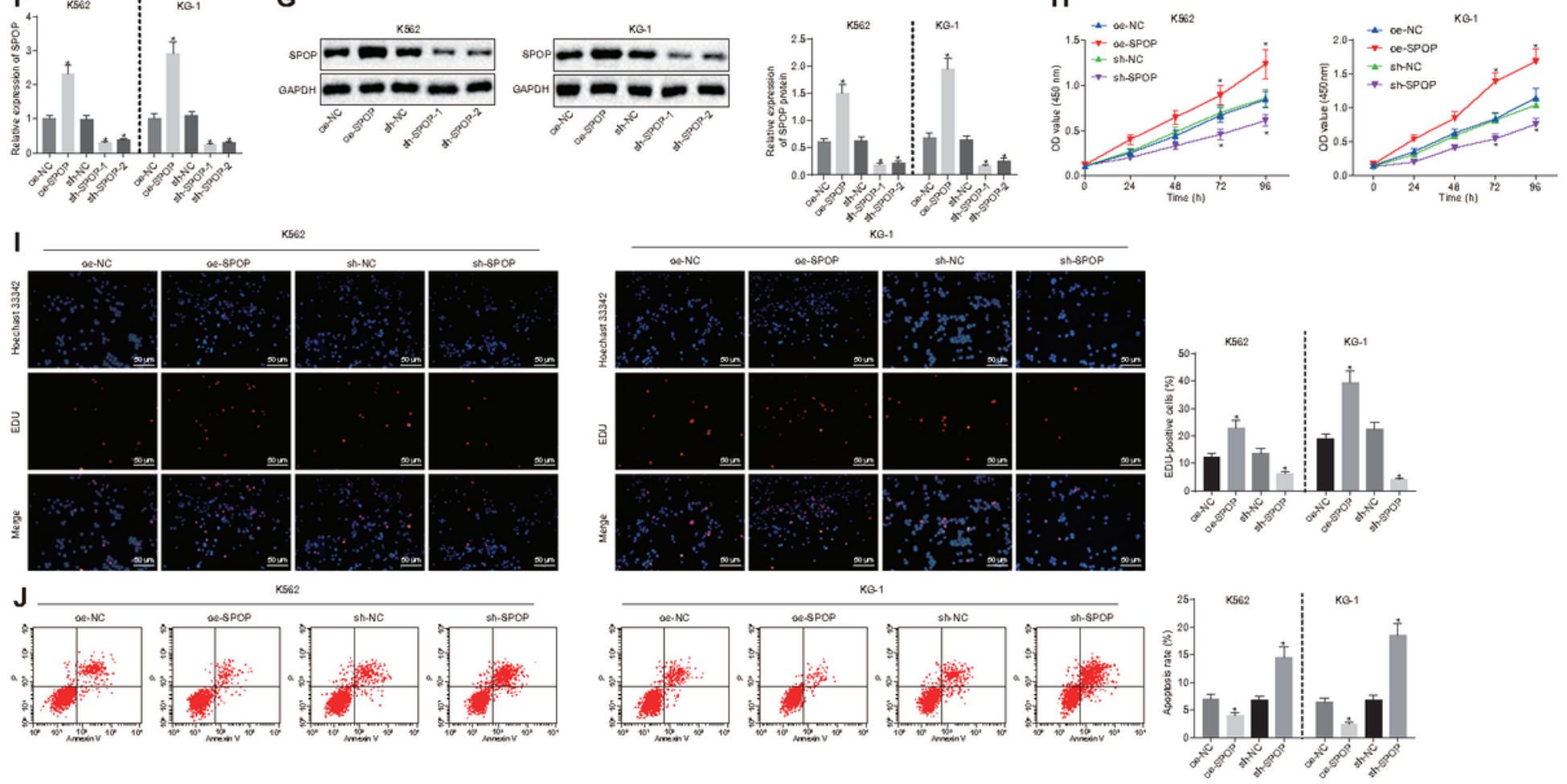

Figure 1 
SPOP overexpression accelerates proliferation and inhibits apoptosis of AML cells. A. The expression of SPOP in AML in the GEPIA database (http.//gepia.cancer-pku.cn/index.html). Red color indicates the leukemia sample and gray color indicates the normal sample. B. Statistical analysis of SPOP mRNA expression in clinical samples detected by qRT-PCR. C. Immunoblots and quantitation of SPOP protein expression in clinical samples detected by Western blot assay. D. Statistical analysis of SPOP mRNA expression in AML cell lines and PBMC cell line detected by qRT-PCR. E. Immunoblots and quantitation of SPOP protein expression in AML cell lines and PBMC cell line detected by Western blot assay. F. qRT-PCR detection of efficiency of overexpressing or silencing SPOP in K562 and KG-1 cells. G. Western blot detection of efficiency of overexpressing or silencing SPOP in K562 and KG-1 cells. H. Quantitative analysis of viability of K562 and KG-1 cells detected by CCK-8 assay after different treatments. I. Microscopic views and quantitative analysis of K562 and KG-1 cell proliferation after different treatments by EdU assay $(\times 200)$. J. flow cytometric data and quantitative analysis of K562 and KG-1 cell apoptosis after different treatments. ${ }^{*} p<0.05$ vs. Normal/oe-NC/sh-NC/PBMC group, respectively. Measurement data were summarized as mean \pm standard deviation. Data comparison between two groups was performed using unpaired $t$ test, and that among multiple groups using one-way ANOVA with Tukey's post hoc test. Statistical analysis in relation to time-based measurements within each group was performed with the use of repeated measures ANOVA with Bonferroni's post-hoc test. The experiment was repeated three times.

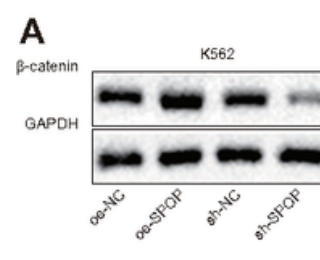

B

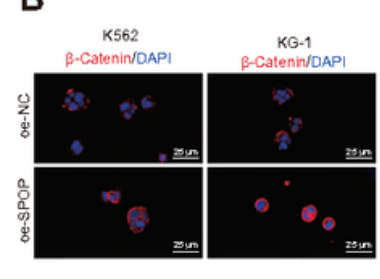

C

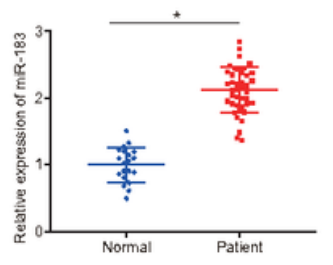

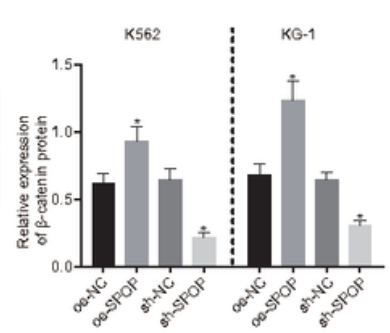

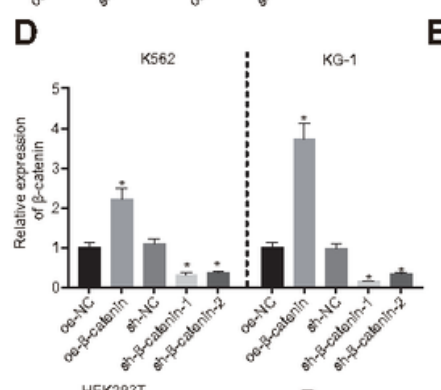

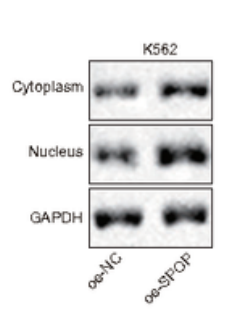

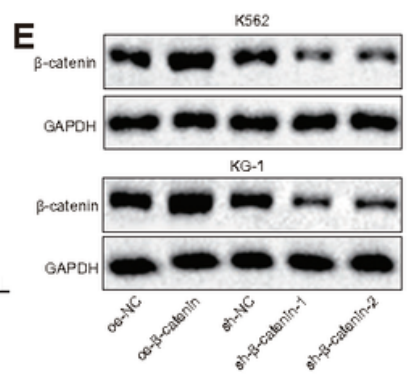

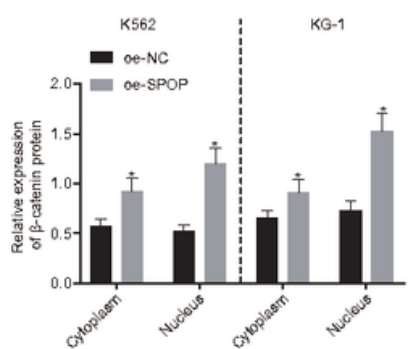

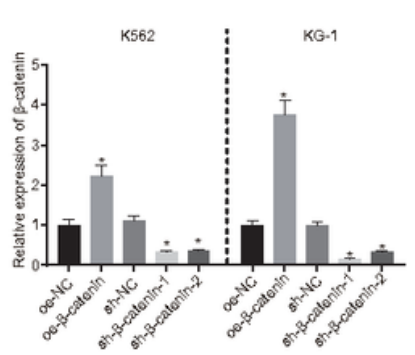

$\mathbf{F}$
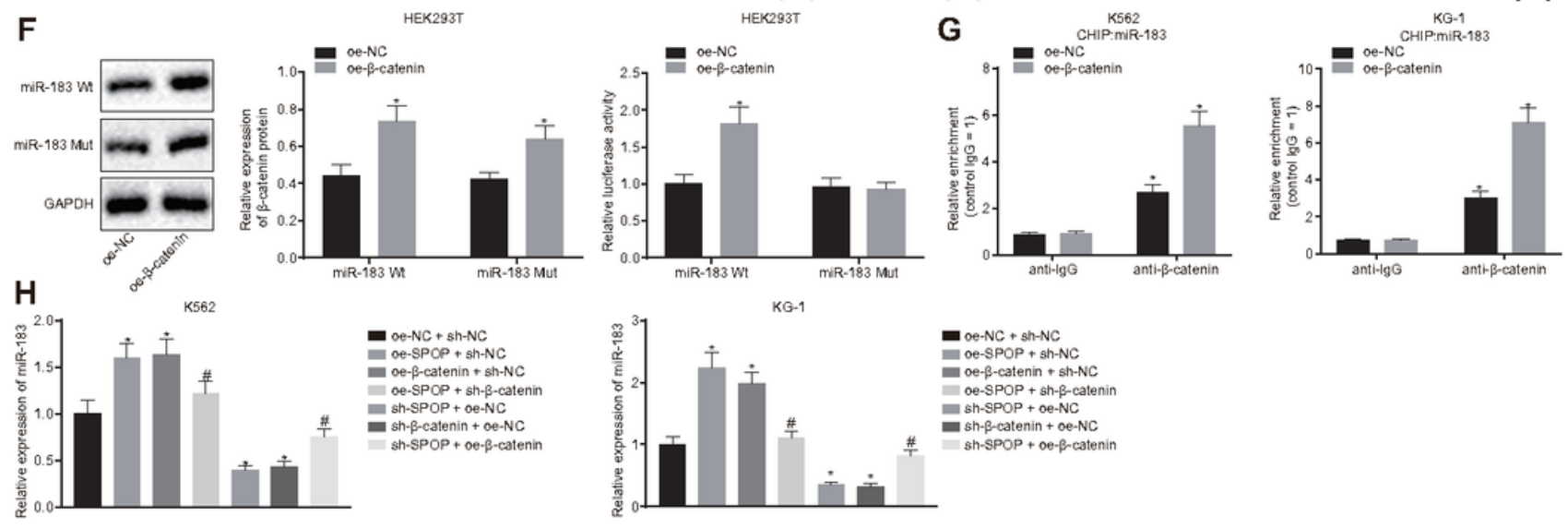

- oe-NC+ sh-NC oe- -3 -catenin + sh- $-\mathrm{NC}$ oe-SPOP + sh- - - catenin 든 sh-SPOP + oe-NC sh-B-catenin + oe-NC

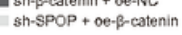

Figure 3 
SPOP accelerates $\beta$-catenin protein expression and nuclear translocation to elevate miR-183 expression. A. Western blot analysis of $\beta$-catenin protein expression in K562 and KG-1 cells, cytoplasm and nucleus after different treatments. B. Immunofluorescence assay of $\beta$-catenin distribution in nucleus and cytoplasm (× 400). C. Quantitative analysis of miR-183 expressions in peripheral blood of clinical samples detected by qRT-PCR. D. Quantitative analysis of efficiency of overexpressing and silencing $\beta$ catenin in K562 and KG-1 cells as detected by qRT-PCR. E. Western blot assay to detect efficiency of overexpressing and silencing $\beta$-catenin in K562 and KG-1 cell. F. Western blot assay to detect $\beta$-catenin expression in HEK293T cells and dual luciferase report assay to detect binding of $\beta$-catenin to miR-183 promoter region. G. CHIP assay to detect $\beta$-catenin enrichment in miR-183 promoter region. H. qRT-PCR to detect miR-183 expression and quantitative analysis. ${ }^{*} \mathrm{p}<0.05$ vs. Normal/oe-NC/sh-NC/oe-NC + sh-NC group. \# $\mathrm{p}<0.05$ vs. oe-SPOP + sh-NC group and sh-SPOP + oe-NC group. Data comparison between two groups was performed using unpaired $t$ test, and that among multiple groups using one-way ANOVA with Tukey's post hoc test. The experiment was repeated three times. 


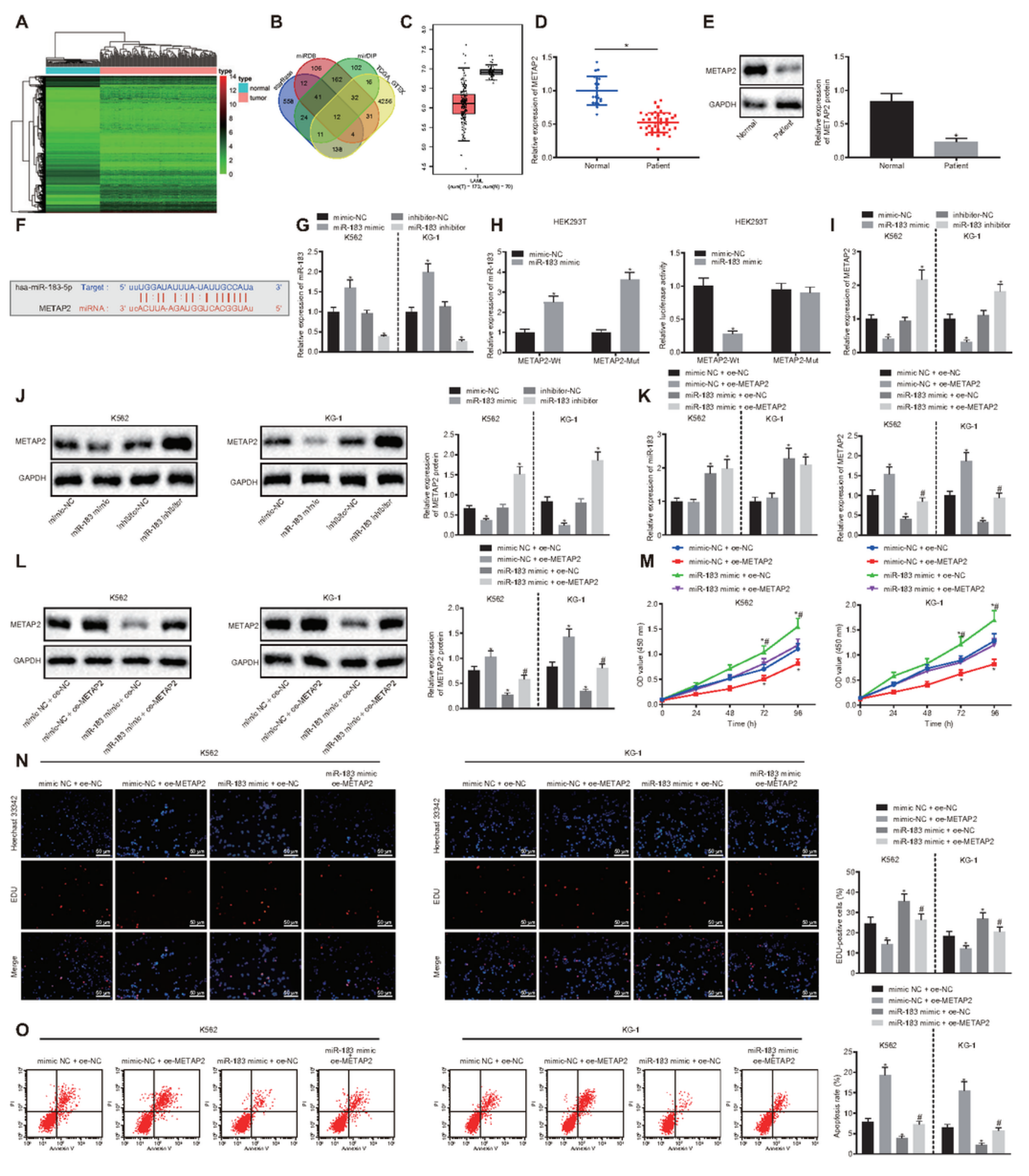

Figure 6

miR-183 overexpression expedites the proliferation and halts apoptosis of AML cells by targeting METAP2. A. The screened differentially expressed mRNAs in AML samples through conjoint analysis of TCGA and GTEX databases. The X-axis represents the sample number and the $Y$-axis represents the mRNA. Each small square in the Figure represents an mRNA in a sample. B. Prediction of target mRNAs in the downstream of miR-183. The four ellipses in the Figure represent the first 800,400 , and 400 genes 
predicted by the starBase (http.//starbase.sysu.edu.cn/index. php), miRDB (http.//mirdb.org), and mirDIP database (http.//ophid.utoronto.ca/mirDIP/index.jsp\#r), respectively. The TCGA and GTEX databases are employed to screen the first 4500 genes that are down-regulated in AML samples. The middle part represents the intersection of the four sets of data. C. The expression of METAP2 in AML in the GEPIA database, red is the AML sample, and gray is the normal sample. D. The qRT-PCR detection of METAP2 expression in clinical samples. E. Western blot detection of protein bands and quantitative analysis of METAP2 expression in clinical samples. F. The binding sites between miR-183 and METAP2 predicted by Starbase database. G. Quantitative analysis of efficiency of overexpressing and inhibiting miR-183 in K562 and KG- 1 cells as detected by qRT-PCR. $\mathrm{H}$. The qRT-PCR detection of miR-183 expression in HEK293T cells and dual luciferase report assay to assess the binding of miR-183 to METAP2. I. The qRTPCR detection of METAP2 mRNA expression in KG-1 and K562 cells after different treatments. J. Western blot detection of METAP2 protein bands and quantitative analysis in KG-1 and K562 cells after different treatments. K. The qRT-PCR detection of miR-183 and METAP2 expression in KG-1 and K562 cells after different treatments. L. Western blot detection of METAP2 protein bands and quantitative analysis in KG1 and K562 cells after different treatments. M. CCK-8 assay of viability of K562 and KG-1 cells after different treatments. N. EdU assay $(\times 200)$ of cell proliferationof K562 and KG-1 cells after different treatments. O. Flow cytometry to detect K562 and KG-1 cell apoptosis and quantitative analysis after different treatments. * $p<0.05$ vs. Normal/inhibitor-NC/mimics-NC/mimics-NC + oe-NC group, respectively, $\# p<0.05$ vs. miR-183 mimics + oe-NC group. Measurement data were summarized as mean \pm standard deviation. Data comparison among multiple groups was performed using one-way ANOVA with Tukey's post hoc test. Statistical analysis in relation to time-based measurements within each group was performed with the use of repeated measures ANOVA with Bonferroni's post-hoc test. The experiment was repeated three times 


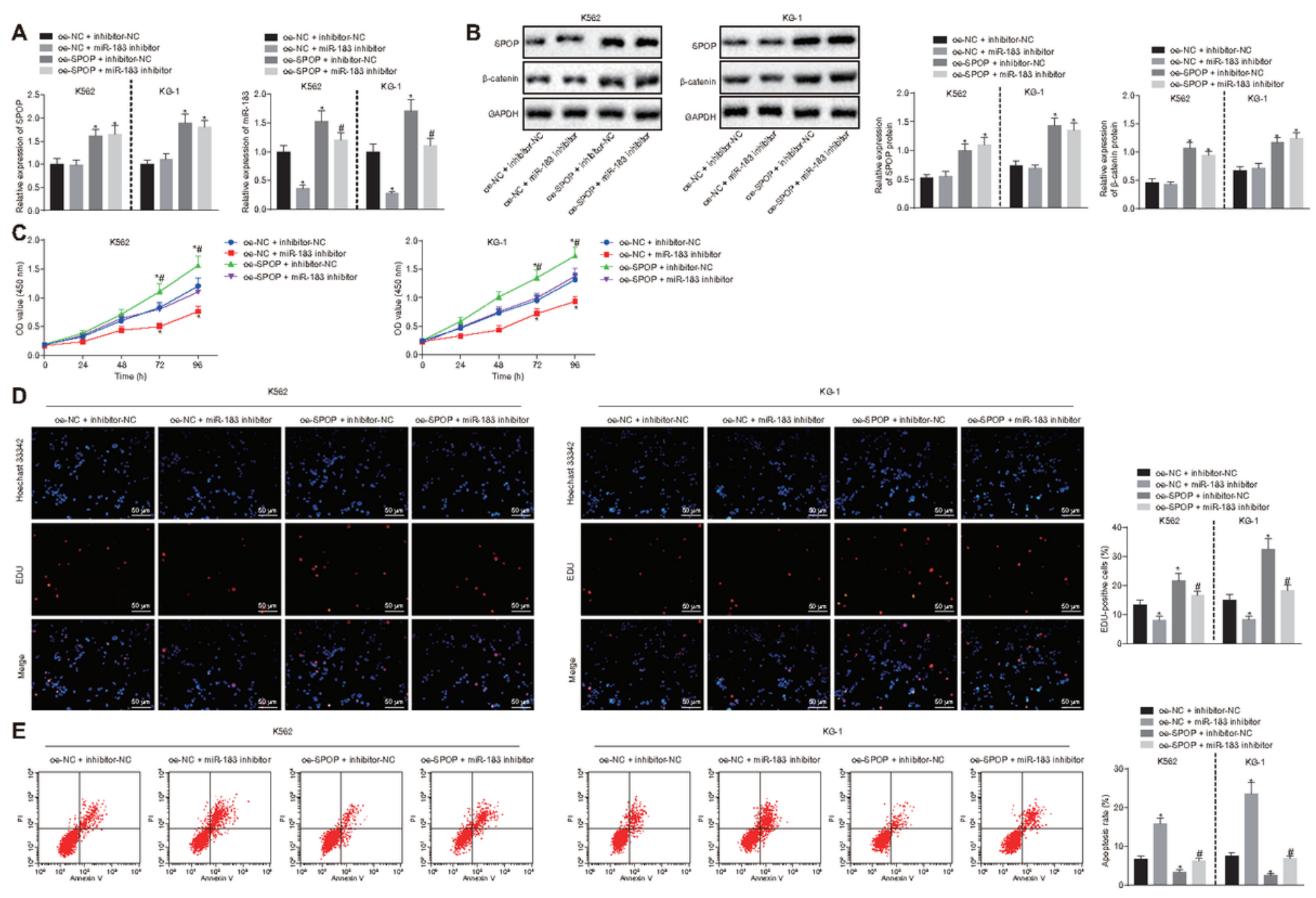

Figure 8

MiR-183 inhibition represses SPOP-induced proliferation of AML cells. A. qRT-PCR detection of SPOP and miR-183 expression in K562 and KG-1 cells after different treatments. B. Western blot detection of SPOP and $\beta$-catenin expression in K562 and KG-1 cells after different treatments and quantitative analysis. C. Quantitative analysis of viability of K562 and KG-1 cells detected by CCK-8 assay after different treatments. D. Microscopic views and quantitative analysis of K562 and KG-1 cell proliferation after different treatments by EdU assay (× 200). E. flow cytometric data and quantitative analysis of K562 and KG-1 cell apoptosis after different treatments. * $p<0.05$ vs. oe-NC + inhibitor-NC group, \# $p<0.05$ vs. oeSPOP + inhibitor-NC group. Measurement data were summarized as mean \pm standard deviation. Data comparison among multiple groups was performed using one-way ANOVA with Tukey's post hoc test. Statistical analysis in relation to time-based measurements within each group was performed with the use of repeated measures ANOVA with Bonferroni's post-hoc test. The experiment was repeated three times. 


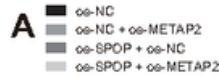
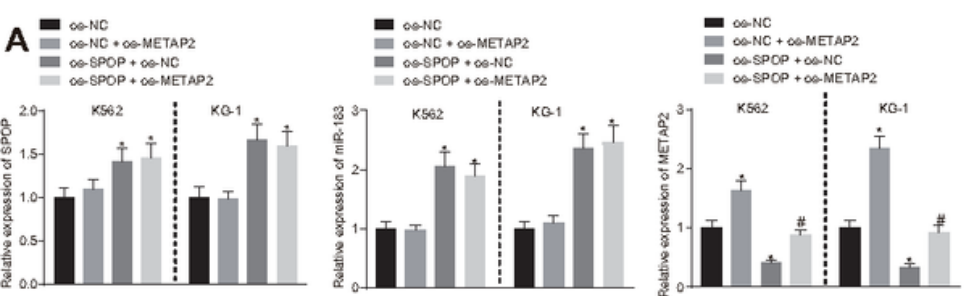

B
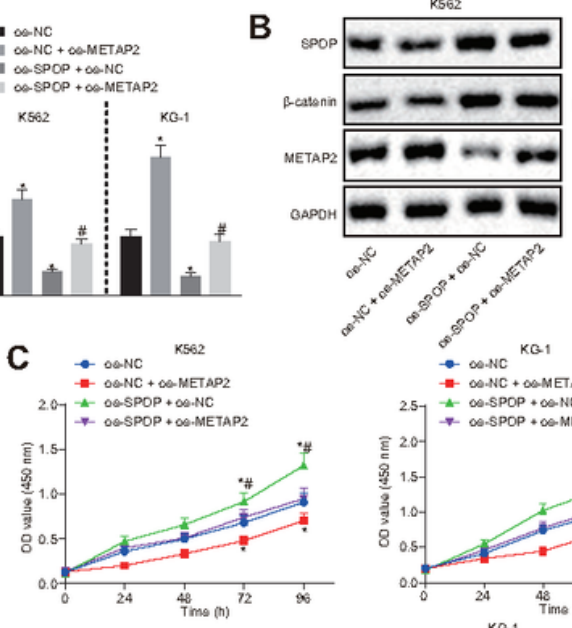

8

- $\infty N C$

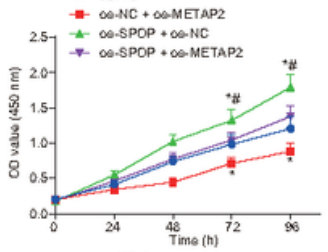

Ke
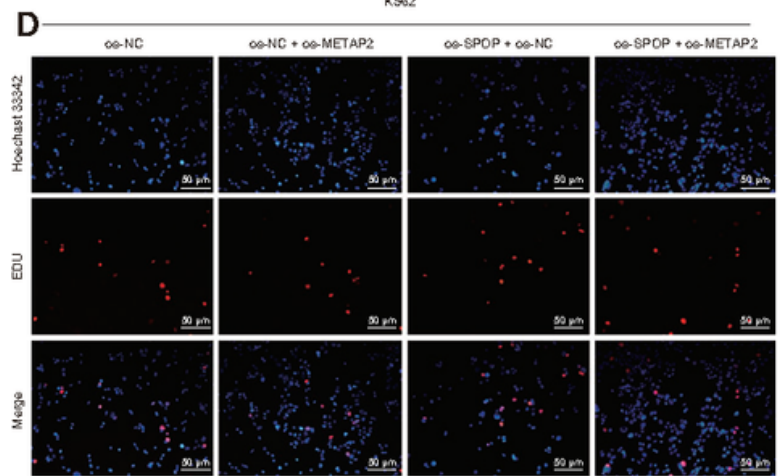

KS

E
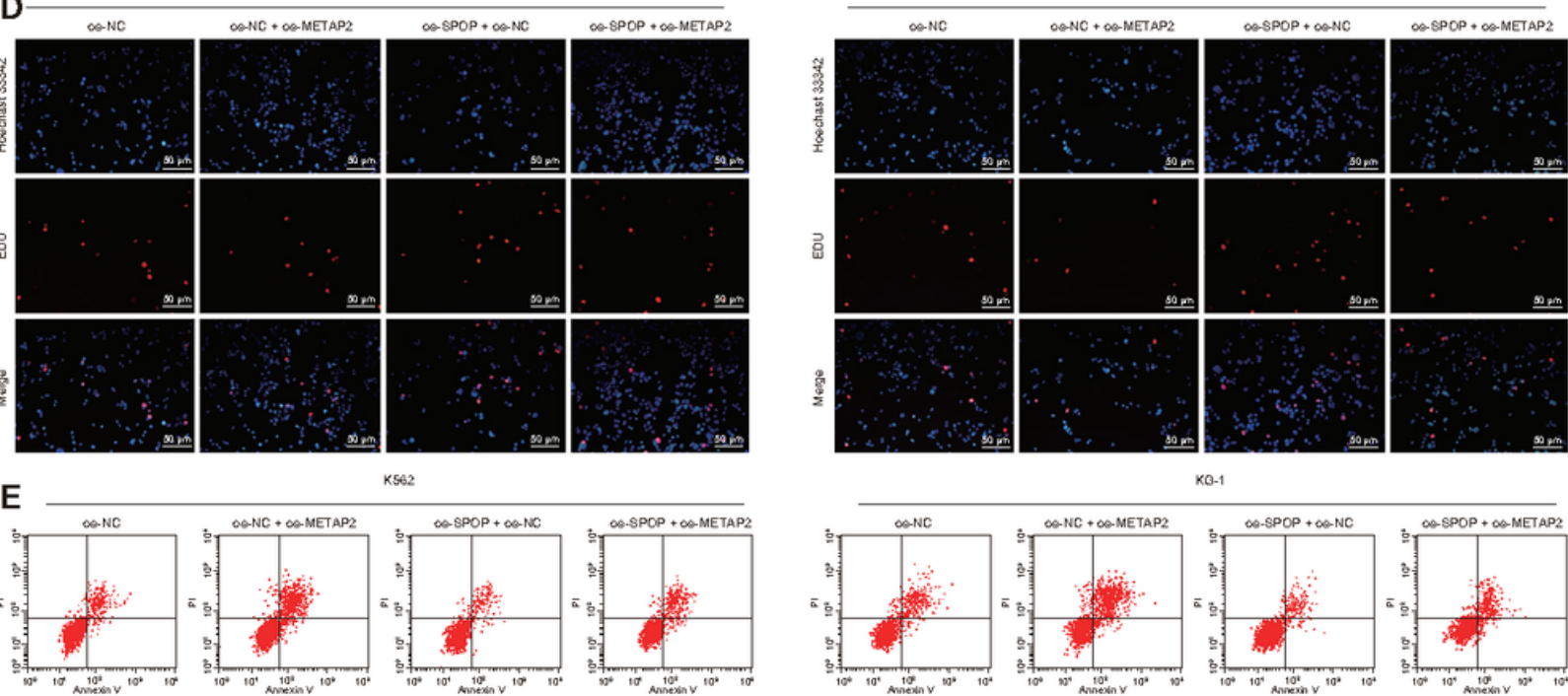

к०-
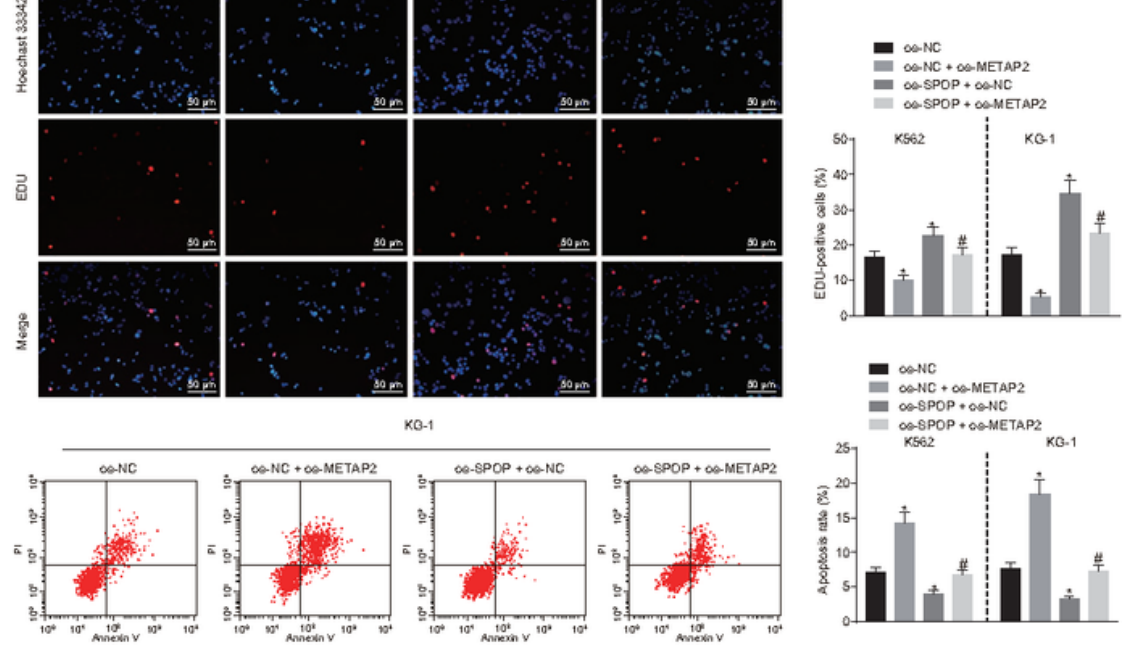

Figure 10

METAP2 overexpression restricts SPOP-induced proliferation of AML cells. A. qRT-PCR detection of SPOP, miR-183 and METAP2 expression in K562 and KG-1 cells after different treatments. B. Western blot detection of SPOP, $\beta$-catenin and METAP2 protein expression in K562 and KG-1 cells after different treatments. C. Quantitative analysis of viability of K562 and KG-1 cells detected by CCK-8 assay after different treatments. D. Microscopic views and quantitative analysis of K562 and KG-1 cell proliferation after different treatments by EdU assay $(\times 200)$. E. flow cytometric data and quantitative analysis of K562 and KG-1 cell apoptosis after different treatments. ${ }^{*} p<0.05$ vs. oe-NC group, \# $p<0.05$ vs. oe-SPOP + oe-NC group. Measurement data were summarized as mean \pm standard deviation. Data comparison among multiple groups was performed using one-way ANOVA with Tukey's post hoc test. Statistical analysis in relation to time-based measurements within each group was performed with the use of repeated measures ANOVA with Bonferroni's post-hoc test. The experiment was repeated three times. 

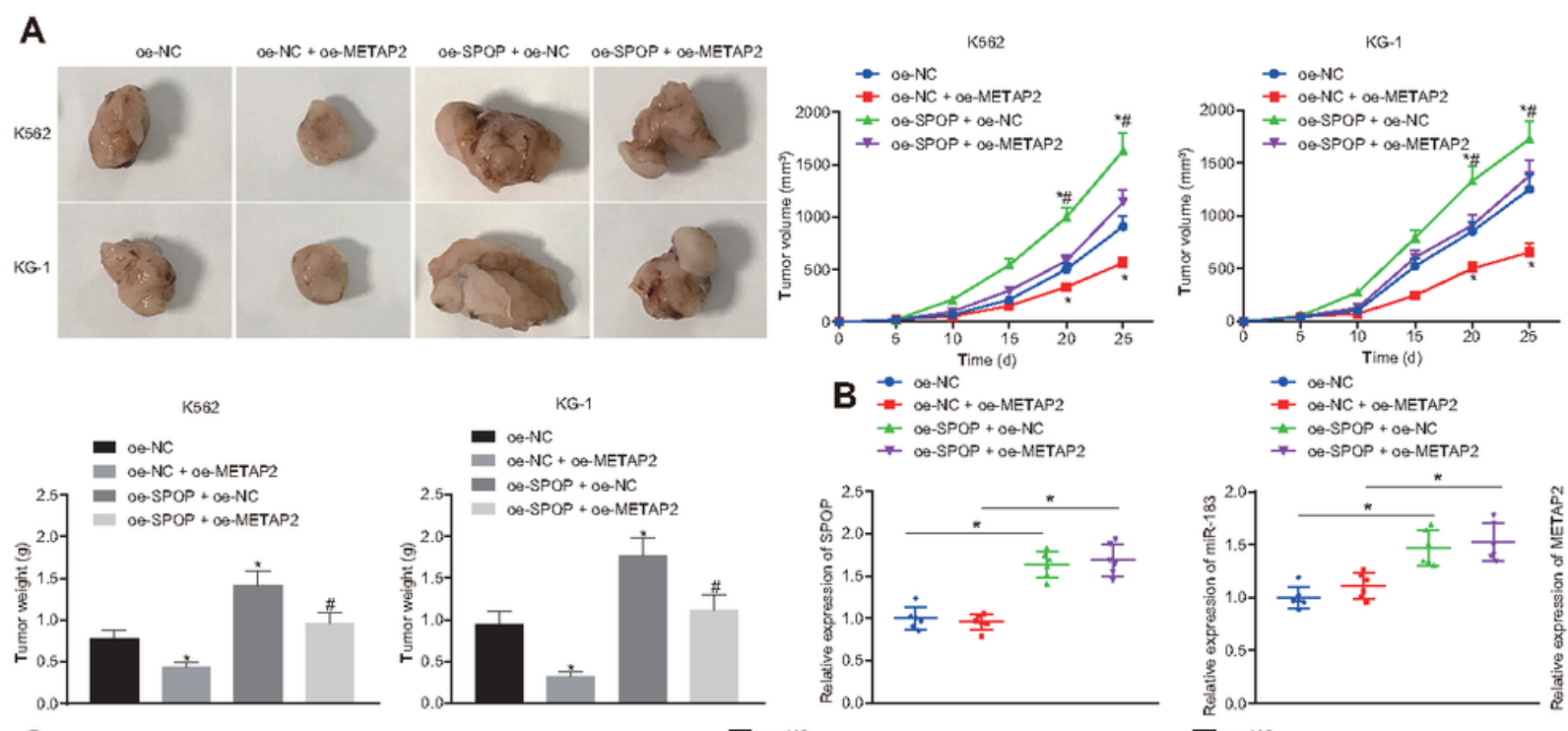

$\Rightarrow$-NC +
$\neq$-METAP2
$\neq$-SPOP + oe-NC
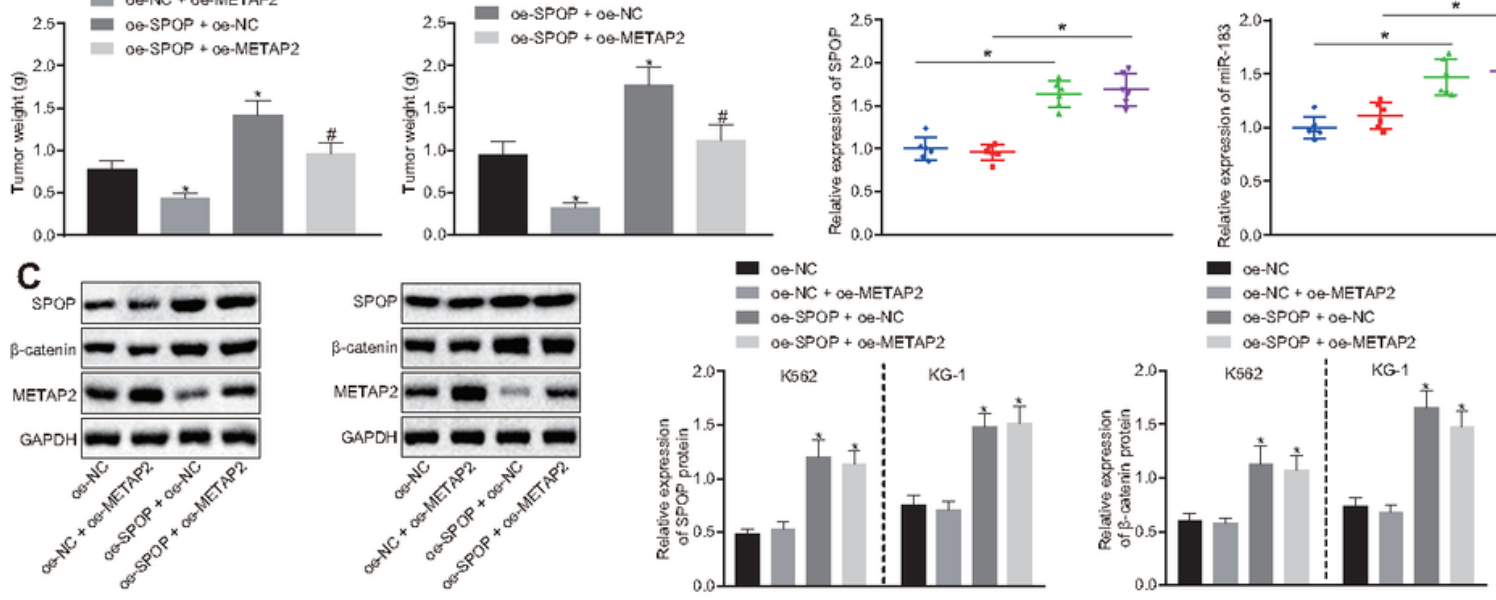

D
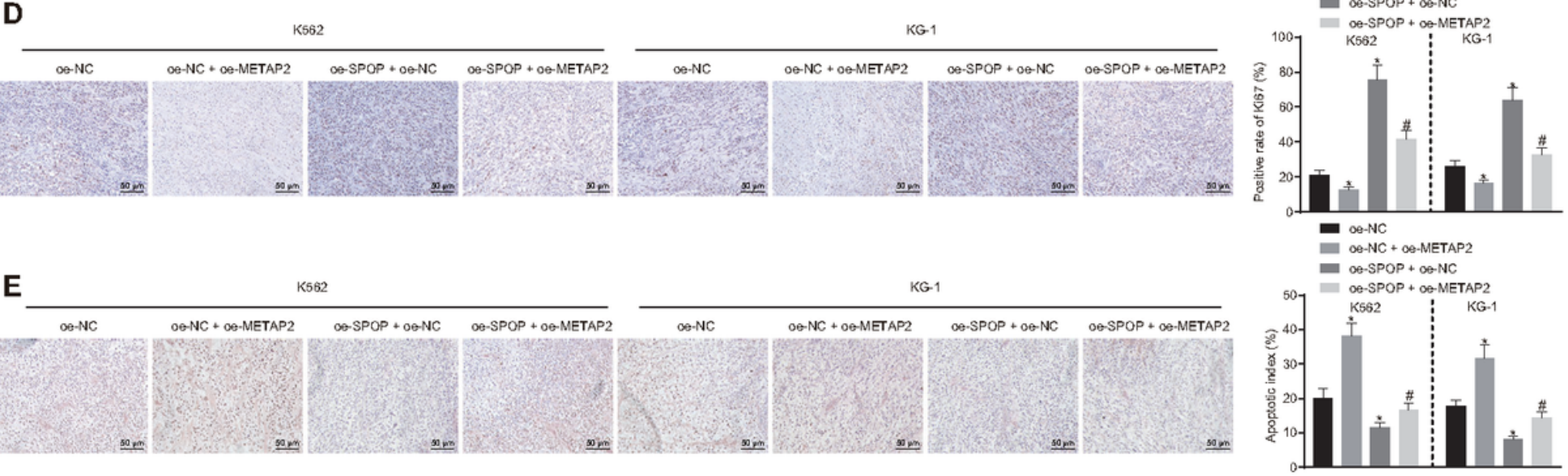

E

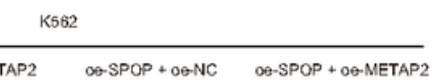

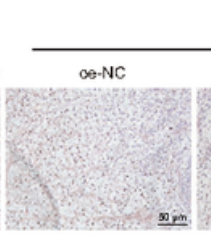

KG-1

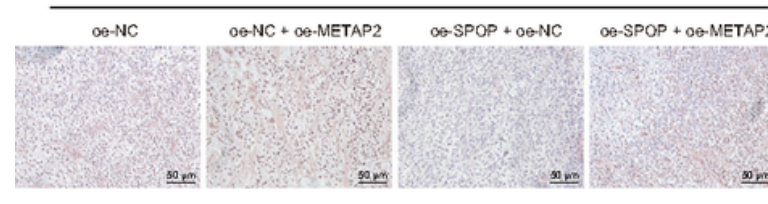

oQ-NC + oe-METAP2

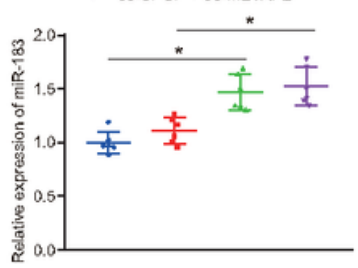

$\rightarrow \infty-N C$

- o-NC + oe-METAP2

\# $\infty$ e-SPOP + oe-NC
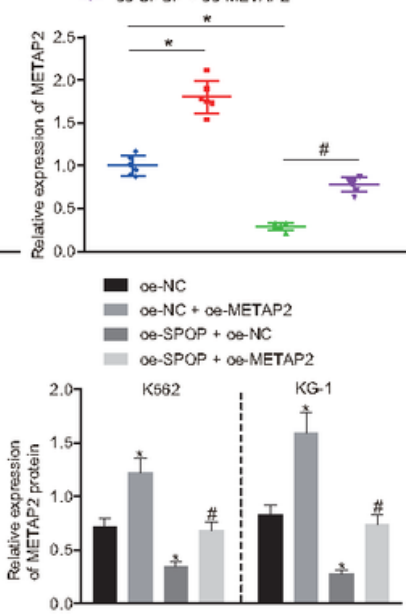

an-NC

ae-NC+ Qe-METAP ae-SPOP+ oe-NC

\section{Figure 11}

SPOP facilitates AML initiation by mediating $\beta$-catenin expression and the miR-183/METAP2 axis. A. Representative images of solid tumors, and quantification of tumor volume and weight. B. qRT-PCR detection of SPOP, miR-183 and METAP2 expression in xenograft tumors of each group. C. Western blot detection of SPOP, $\beta$-catenin and METAP2 protein expression bands and quantitative analysis in xenograft tumors. D. Ki67 immunohistochemical analysis $(\times 200)$ of cell proliferation in xenograft tumors and quantitative analysis. E. TUNEL staining $(\times 200)$ for cell apoptosis in xenograft tumors and quantitative analysis. $n=6$. ${ }^{*} p<0.05$ vs. oe-NC group, $\# p<0.05$ vs. oe-SPOP + oe-NC group. Measurement data were summarized as mean \pm standard deviation. Data comparison among multiple groups was performed using one-way ANOVA with Tukey's post hoc test. Statistical analysis in relation to 
time-based measurements within each group was performed with the use of repeated measures ANOVA with Bonferroni's post-hoc test.

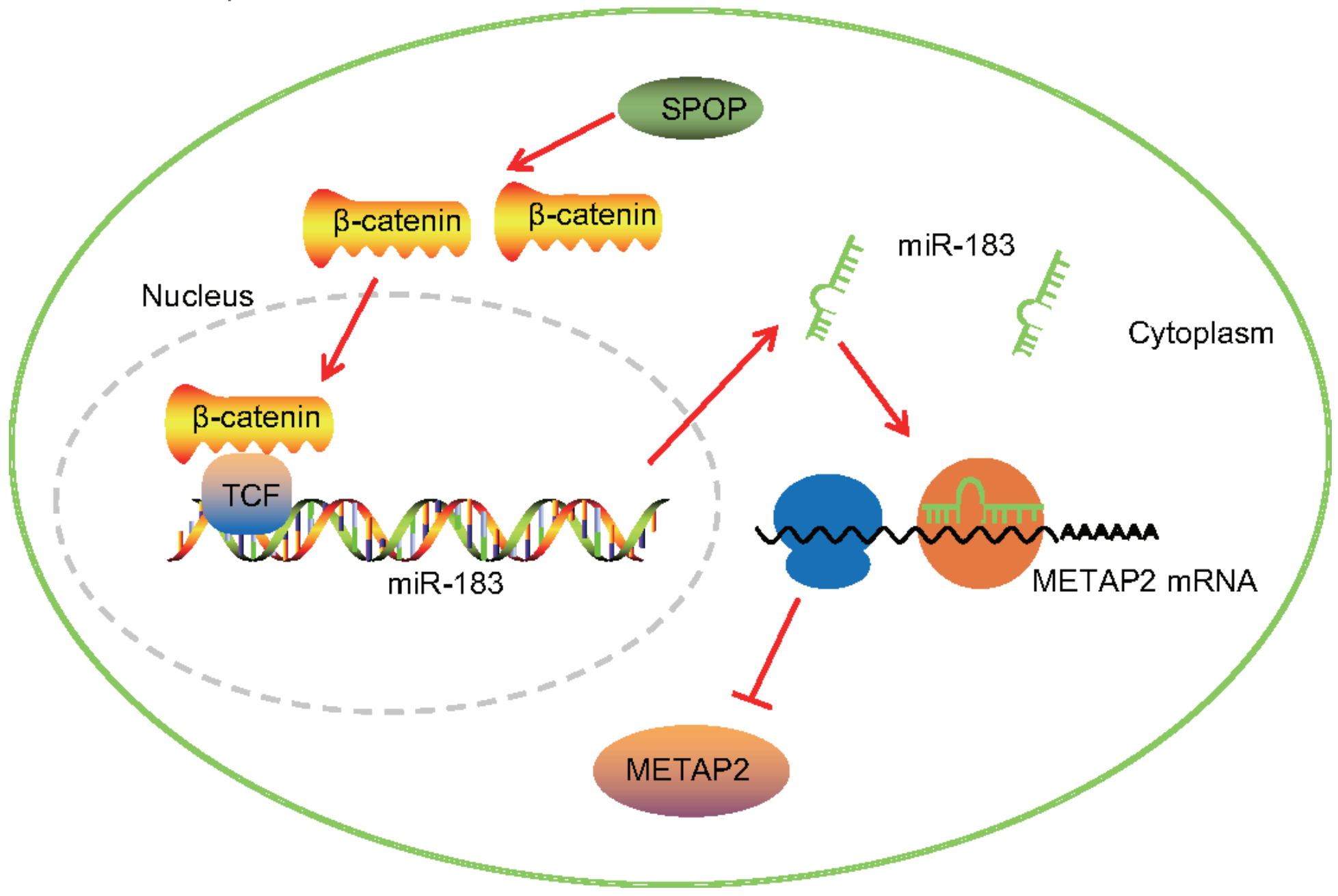

\section{Figure 13}

Schematic representation of the potential molecular mechanisms of SPOP in AML. SPOP is highly expressed in AML cells. SPOP augments $\beta$-catenin cytoplasmic stability and nuclear translocation. Moreover, $\beta$-catenin can bind to the miR-183 promoter region and upregulates miR-183 expression. MiR183 targets and inhibits METAP2 in the cytoplasm, thereby promoting AML cell proliferation and inhibiting apoptosis leading to AML initiation. 